\title{
Acute disseminated encephalomyelitis caused by Epstein- Barr virus infection in an immunocompetent adult woman
}

\author{
Hyunjoo $\mathrm{Oh}^{1}$, Jeong Rae Yoo ${ }^{1}$, SangTaek Heo ${ }^{1, \mathbb{}}$, Jung-Hwan $\mathrm{Oh}^{2}$, Ho Kyu Lee $e^{3}$ \\ Departments of ${ }^{1}$ Internal Medicine, ${ }^{2}$ Neurology, and ${ }^{3}$ Radiology, Jeju National University School of Medicine, \\ Jeju, Republic of Korea
}

\begin{abstract}
Epstein-Barr virus (EBV) infection is common and usually asymptomatic in young infants and children. However, EBV infections in transplant recipients and other immunosuppressed patients can be fatal. EBV-related neurological complications in immunocompetent adults are extremely rare and self-limited. Acute disseminated encephalomyelitis (ADEM) may also follow EBV infection; ADEM is characterized by abrupt onset and rapid progression. We report an immunocompetent adult patient who developed diffuse meningoencephalitis with ADEM-like features caused by EBV infection. A 35-year-old Vietnamese woman was admitted presenting with urinary retention, altered mental status, and paraplegia. PCR of the patient's cerebrospinal fluid showed positive results for EBV. Brain and spine magnetic resonance imaging showed ADEM-like features. She was treated with acyclovir, steroid, and immunoglobulins. We report the case of an immunocompetent adult Vietnamese woman who presented with rapidly progressive diffuse meningoencephalitis associated with EBV infection and was treated with antivirals, corticosteroids, and immunoglobulins.
\end{abstract}

Key words: Acute disseminated encephalomyelitis, Epstein-Barr virus, Acyclovir, Immunocompetent

\section{INTRODUCTION}

Among patients with viral meningoencephalitis, Ebstein-barr virus (EBV) is a common pathogen. ${ }^{1,2)} \mathrm{EBV}$ encephalitis has been frequently reported to be asymptomatic in children, ${ }^{1,3)}$ latent EBV infection is prevalent in more than $90 \%$ of the adult population, and reactivation is associated with other infectious or inflammatory processes in adults. ${ }^{4)}$ Sometimes, EBV infection can be fatal to transplant recipients and immunosuppressed patients. ${ }^{5)}$ However, EBV infection is rarely associated with neurological complications, such as meningitis, encephalitis, meningo-

Received: December 26, 2018; Revised: January 5, 2019; Accepted: January 7, 2019

$>$ Correspondence to : SangTaek Heo

Department of Internal Medicine, Jeju National University School

of Medicine, 102 Jejudaehakno, Jeju-si, Jeju Special Self-Governing

Province, 63243, Korea

Tel: 82-64-717- 1296, FAX: 82-64-717-1131

E-mail: neosangtaek@naver.com encephalitis, myelitis, polyradiculitis, polyradiculomyelitis, acute cerebellar ataxia, and acute disseminated demyelinating encephalomyelitis. ${ }^{2,3,6)}$ The overall incidence of neurological complications of EBV infection has been reported to be $<7 \%{ }^{3,7)}$ Acute disseminated encephalomyelitis (ADEM) is a heterogenous syndrome that is occasionally triggered by an antecedent infection such as influenza or EBV infection. Herein, we report a case of meningoencephalitis with ADEM-like features in an immunocompetent adult, caused by an EBV infection confirmed by the presence of EBV DNA in the cerebrospinal fluid (CSF). The study was approved by the Institutional Review Board of Jeju National University Hospital (JNUH 201806024).

\section{CASE REPORT}

A 35-year old woman initially developed headache, malaise and fever followed by urinary retention 10 days later. 
Table 1. Cerebrospinal fluid analyses of the patient

\begin{tabular}{lccc}
\hline Date of Admission & $\begin{array}{c}\text { White blood cell counts } \\
\text { (Lymphocyte, } \%)\end{array}$ & $\begin{array}{c}\text { Protein } \\
(\mathrm{mg} / \mathrm{dL})\end{array}$ & $\begin{array}{c}\text { Glucose }(\mathrm{mg} / \mathrm{dL}) \\
(\text { Serum glucose, mg/dL) }\end{array}$ \\
\hline Day 1 & $158(100)$ & 70 & $40(95)$ \\
Day 2 & $65(84)$ & 2 & $60(132)$ \\
Day 5 & $410(96)$ & 86 & $51(221)$ \\
Day 47 & $30(98)$ & 28 & $50(106)$ \\
\hline
\end{tabular}
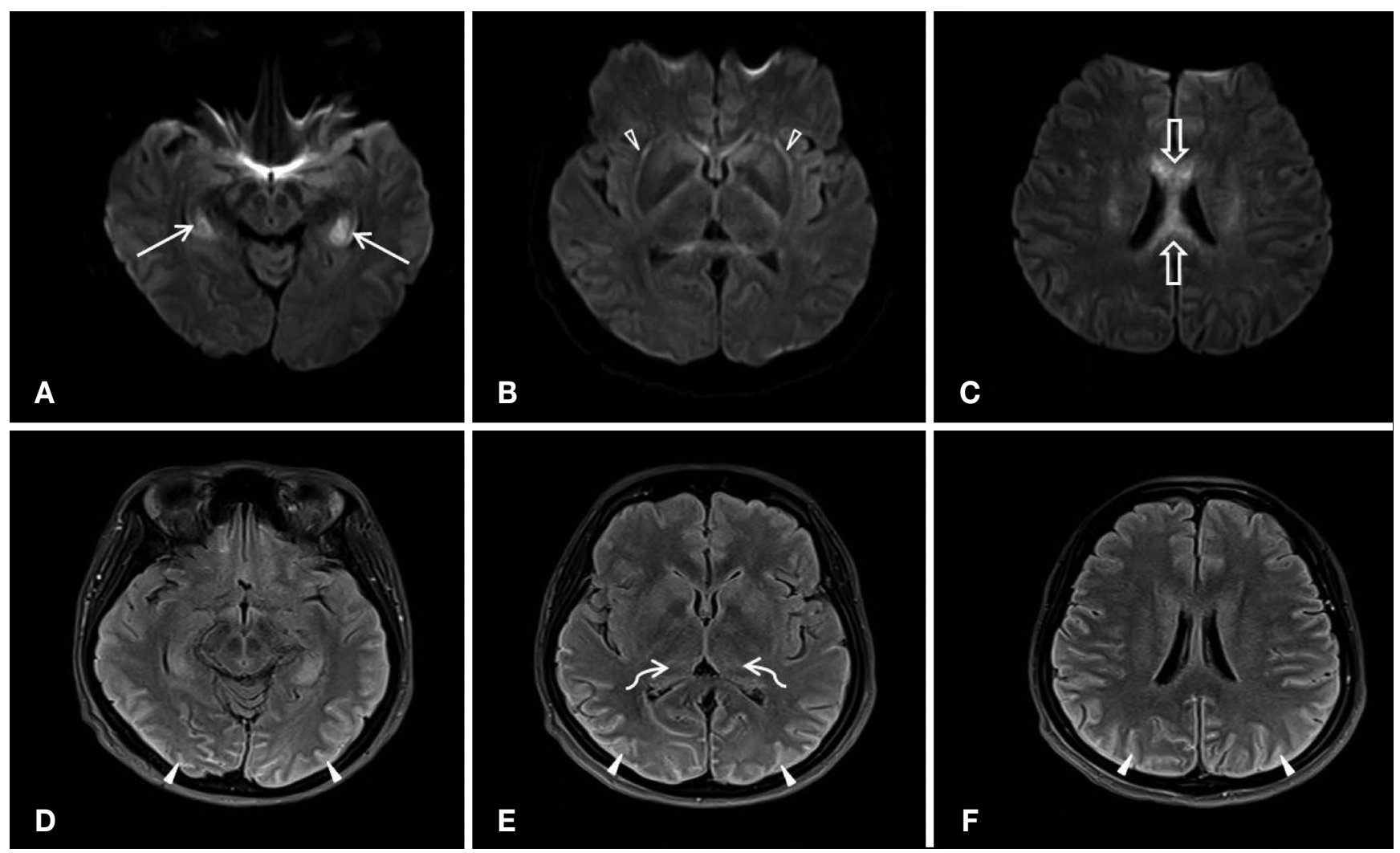

Figure 1. Brain MRI on admission day 2. A, B, C: Diffusion weighted images show areas of restricted diffusion in both hippocampi (arrows), lentiform nuclei, external capsules (open arrow-heads), internal capsules, corona radiata, and corpus callosum (open arrows) in symmetric fashion. D, E, F: T2-FLAIR images show high signal intensity in both thalami (curved arrows) and gyri of both parieto-occipital lobes (closed arrow-heads) as well as above locations.

She was admitted presenting with urinary retention to the urology department of the local clinic. She was Vietnamese and had no significant past medical history or family history. On the second day of admission at the local clinic, she developed decreased level of consciousness. A lumbar puncture was performed and CSF analysis revealed a white blood cell count of $158 / \mathrm{mm}^{3}$ (lymphocytes, $100 \%$ ), protein level of $70 \mathrm{mg} / \mathrm{dL}$, and glucose level of $40 \mathrm{mg} / \mathrm{dL}$ (serum glucose level, $95 \mathrm{mg} / \mathrm{dL}$ ) (Table 1). The initial brain magnetic resonance imaging (MRI) revealed multiple sulcal high signal changes and gyral swelling in both high fronto-parietal regions (Fig. 1). She was transferred to our tertiary hospital emergency department. On neurological examination, she demonstrated drowsy mental status, neck stiffness, and weakness of both legs (Medical Research Council (MRC) grade 0 or 1). Laboratory analysis showed the following results: white blood cell count: $15.3 \times 10^{9} / \mathrm{L}$ (reference range $4.0 \sim 10.0 \times 10^{9} / \mathrm{L}$ ); hemoglobin $(\mathrm{Hb})$ level: $12.4 \mathrm{~g} / \mathrm{dL}$ (reference range $13 \sim 17 \mathrm{~g} / \mathrm{dL}$ ); and C-reactive protein level: $1.82 \mathrm{~g} / \mathrm{dL}$ (reference range $0 \sim 0.30 \mathrm{~g} / \mathrm{dL}$ ). Antinuclear antibody test was negative. Her electrocardiogram showed bradycardia (heart rate, 59/min) with sinus 

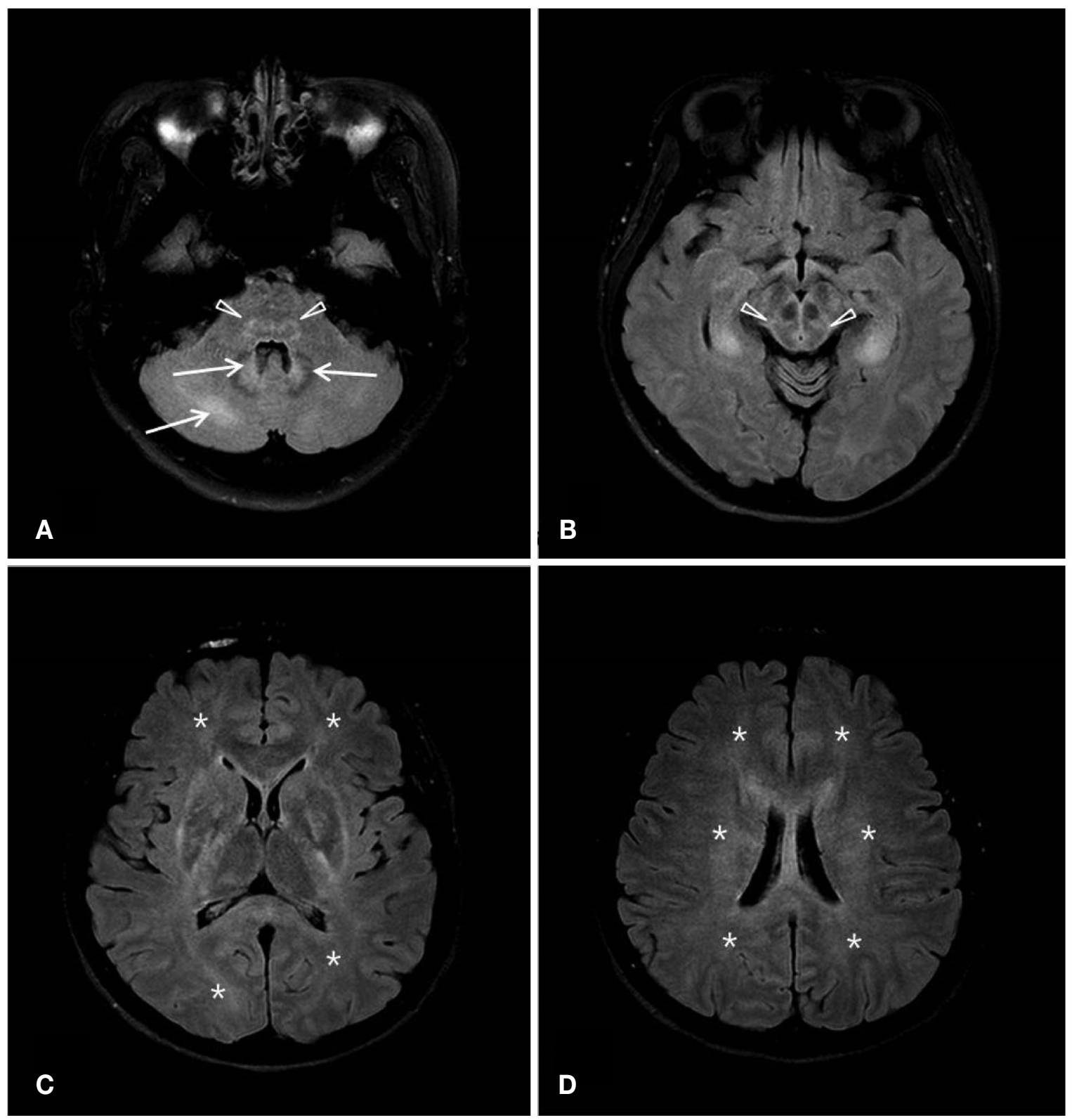

Figure 2. Brain MRI on admission day 8. A, B, C, D: T2-FLAIR images show interval progression of high signal intense lesions in extent and intensity. In addition of the lesions seen on the admission day 2, there was development of lesions of high signal intensity in the cerebellum(arrows), brain stem (open arrow-heads), and centrum semiovale (stars) on both sides.

rhythm and chest X-ray showed mild peribronchial infiltration in right lung field. Her second CSF analysis revealed a white blood cell count of $65 / \mathrm{mm}^{3}$ (polymorphonuclear leukocytes, $3 \%$; lymphocytes, $84 \%$; monocytes, $12 \%$; eosinophils, $1 \%$ ), protein level of $2 \mathrm{mg} / \mathrm{dL}$, and glucose level of $60 \mathrm{mg} / \mathrm{dL}$ (serum glucose level, $132 \mathrm{mg} / \mathrm{dL}$ ) (Table 1). A polymerase chain reaction (PCR) of the patient's CSF was carried out for the detection of herpes simplex virus type 1 and 2, enterovirus, EBV, cytomegalovirus, varicella zoster,
Mycobacterium tuberculosis, and non-tuberculous mycobacteria. Intravenous vancomycin ( $20 \mathrm{mg} / \mathrm{kg}$ every $12 \mathrm{~h}$ ), and ceftriaxone ( $2 \mathrm{~g}$ every $12 \mathrm{~h})$, and acyclovir $(10 \mathrm{mg} / \mathrm{kg}$ every 8 hours) were administered initially to the patient at the emergency department. On the second day of admission, the patient showed rapid worsening of consciousness to stupor and experienced generalized seizures and severe hypercapnic respiratory failure. Antiepileptic drugs were added to her treatment regimen and mechanical ventila- 
tion was provided. Electroencephalography (EEG) showed generalized slow pattern without epileptiform discharge. Brain MRI revealed areas of restricted diffusion in the corpus callosum, hippocampi, and external capsules in a symmetric fashion on diffusion-weighted images (Fig. 1). Fluid-attenuated inversion recovery (FLAIR) images revealed increased T2 high signal intensity in the both lentiform nuclei and parieto-occipital lobes in addition to the above anatomic locations (Fig. 1).

From the fourth day of admission, her consciousness gradually recovered but flaccid paraplegia continued. Although we strongly suspected viral meningoencephalitis, autoimmune encephalitis or ADEM could not be completely ruled out. She was tested for the autoimmune synaptic encephalitis antibodies. Intravenous methylprednisolone $(1,000 \mathrm{mg} /$ day) and intravenous immunoglobulin (IVIG) $(0.4 \mathrm{~g} / \mathrm{kg} / \mathrm{day})$ for possible viral or autoimmune meningoencephalitis were administered for five days in addition to the intravenous acyclovir and other antibiotics.

The CSF PCR result was reported as only positive for EBV DNA. Viral culture was not performed and the bacterial and fungal cultures were negative. Serological tests for EBV showed that EBV viral capsid antigen (VCA) IgM, EBV early antigen (EA) IgM and EBV EA IgG were absent whereas EBV nuclear antigen (EBV-EBNA) IgG and EBV VCA IgG were present. Autoimmune synaptic encephalitis antibodies (NMDAR, AMPA1, AMPA2, LGI1, CASPR2, and GABA-B) and classic paraneoplastic syndrome antibodies (Hu, Yo, Ri, Ma2, CV2/CRMP5, Amphiphysin, Recoverin, SOX1, and Titin) were not detected. Empiric vancomycin and ceftriaxone were discontinued after the final negative CSF results for other microorganisms were confirmed, but intravenous acyclovir was administered for 14 days. Brain T2-weighted MRI showed interval progression of high signal intensity in extent and intensity, in both the supratentorial and infratentorial regions of the brain (Fig. 2). Evaluation of spine MRI to assess paraplegia and urinary retention was performed 8 days after admission and showed diffuse swelling of the spinal cord and symmetrical high signal intensity in the central column on T2-weighted images (Fig. 3). She gradually recovered and was discharged after two months of admission with a Glasgow coma score of 15 ; she was conscious and fully oriented, but still had flaccid paraplegia and urinary retention. At the 3-month follow-up, her urinary retention had resolved and she could walk using a cane. Follow-up MRI revealed that all signal intensities of previous lesions were normalized.

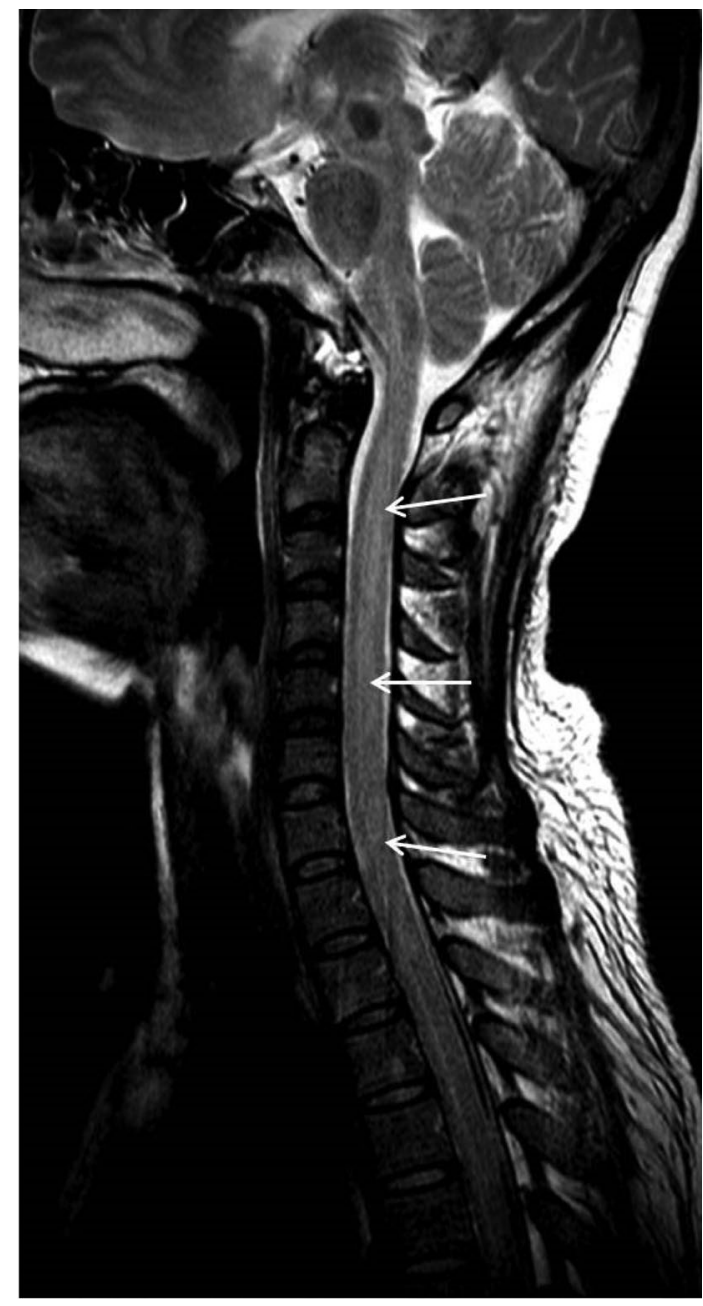

Figure 3. Spine MRI on admission day 8. T2-weighted image shows diffuse swelling of the cervical cord with diffuse high signal intensity in the central column (arrows).

\section{DISCUSSION}

We encountered a case of EBV infection causing meningoencephalitis in an immunocompetent adult. EBV DNA was identified in the patient's CSF using PCR, and CSF analysis was highly suggestive of viral meningitis. Her MRI results were consistent with encephalitis and spinal myelitis. Reports of incidence of neurological complications following symptomatic EBV infection in adults have been rare. ${ }^{3,7)}$ Most neurological complications of EBV infections have been reported in children under 5 or over 10 years of age. ${ }^{3)}$ In the study by Doja et al., evidence of acute EBV infection was found in $9.7 \%$ of children admitted with acute encephalitis. ${ }^{3)}$ EBV is also known to cause severe diseases in immunocompromised patients such as 
those with late-stage acquired immunodeficiency syndrome (AIDS) and those who have undergone translplant. ${ }^{8)}$ Some cases involving EBV encephalitis with asymmetric central nervous system (CNS) involvement in immunocompetent patients have been reported. ${ }^{9)}$ The prognosis of CNS involvement in EBV infection varies from complete recovery to death. In their literature review, Abul-Kasim et al. reported that EBV infection involving the brain stem had a less favorable prognosis than that of EBV infection involving the cerebral hemisphere, thalamus, and limbic system. ${ }^{10)}$ Our patient's MRI showed diffuse and symmetric involvement of the thalamus, basal ganglia, corpus callosum, hippocampus and spinal cord, which may relate to the residual neurological deficit. This case demonstrated that EBV infection can affect the CNS and cause neurologic sequelae in immunocompetent patients.

The pathophysiology of EBV-induced neurological injury remains unclear. Primary inflammation due to direct viral invasion, secondary autoimmune reaction or both have been suggested as the possible mechanisms by which EBV cause encephalitis. ${ }^{2)}$ In addition, CSF PCRs showing positive results for EBV have been associated with positive results for other pathogens, and may reflect reactivation of latent EBV in lymphocytes that enter the CNS as a result of an unrelated infectious or inflammatory process. ${ }^{11)}$ Serological and molecular testing of the patient showed negative results for other viruses; furthermore, bacterial cultures were also negative and no neoplastic cells were detected. The serology test results indicated that the patient had been previously infected with EBV, and therefore, we suspected that reactivation of EBV had occurred in response to a secondary autoimmune reaction.

With regards to the differential diagnosis, distinction from ADEM may be difficult because neurological symptoms and signs of our patient were not distinguishable from ADEM. ADEM is usually a monophasic inflammatory demyelinating disorder of the CNS that occurs within days to weeks of a viral illness or vaccination. The preceding infection is typically a benign upper respiratory tract infection or a non-specific febrileillness. ${ }^{12)}$ Systemic viral infections proceeding ADEM has been predominantly detected in pediatric patients, ${ }^{13)}$ whereas it has been rarely reported in adult patients. ${ }^{14)}$ In patients with ADEM, diffuse or multifocal asymmetrical lesions throughout the white matter were observed on T2-and FLAIR-weighted MRI sequences. ${ }^{12,15)}$ Our patient tested negative for all specific autoimmune antibodies; nevertheless, her MRI results showed symmetric diffuse multifocal white matter lesions in the brain and spinal cord involvement like ADEM.
Therefore, the possibility of concurrent presentation of ADEM in our patient could not be excluded completely.

Although there is no specific treatment against EBV, antivirals such as acyclovir and corticosteroids have been prescribed for treatment of EBV-related complications in immunocompetent adults. ${ }^{8)}$ Acyclovir inhibits replication of EBV in vitro and has been used in some cases of CNS disease; however, the efficacy of acyclovir in EBV infection has not been established and there is no consensus on the use of antiviral medications for EBV infection. ${ }^{48}$ In the present case, intravenous acyclovir, high-dose corticosteroid pulse and IVIG were administered to the patient. Corticosteroids and IVIG can be used for not only EBV infection but also for immune-mediated CNS diseases, such as ADEM. ${ }^{3,6)}$

In conclusion, we described the case of an immunocompetent adult Vietnamese woman who presented with rapidly progressive diffuse meningoencephalitis associated with EBV infection. Although there have been reports describing CNS involvement in EBV infection in children meningoencephalomyelopathy associated with EBV infection is rare in an immunocompetent adult. Our experience suggests that EBV infection may cause diffuse CNS involvement in spite of the absence of immunodeficiency and can be treated with antivirals, corticosteroids, and IVIG.

\section{REFERENCES}

1. Kumar R, Kumar P, Singh MK, Agarwal D, Jamir B, Khare S, et al. Epidemiological profile of acute viral encephalitis. Indian J Pediatr 2018;85:358-63.

2. Fujimoto H, Asaoka K, Imaizumi T, Ayabe M, Shoji H, Kaji M. Epstein-Barr virus infections of the central nervous system. Intern Med 2003;42:33-40.

3. Doja A, Bitnun A, Ford Jones EL, Richardson S, Tellier R, Petric M, et al. Pediatric Epstein-Barr virus-associated encephalitis: 10-year review. J Child Neurol 2006;21:384-91.

4. Tunkel AR, Glaser CA, Bloch KC, Sejvar JJ, Marra CM, Roos KL, et al. The management of encephalitis: clinical practice guidelines by the Infectious Diseases Society of America. Clin Infect Dis 2008;47:303-27.

5. Lau JSY, Low ZM, Abbott I, Shochet L, Kanellis J, Kitching AR, et al. Epstein-Barr virus encephalitis in solid organ transplantation. New Microbiol 2017;40:212-7.

6. Majid A, Galetta SL, Sweeney CJ, Robinson C, Mahalingam R, Smith J, et al. Epstein-Barr virus myeloradiculitis and encephalomyeloradiculitis. Brain 2002;125:159-65.

7. Hagemann G, Mentzel HJ, Weisser H, Kunze A, Terborg C. Mul- 
tiple reversible MR signal changes caused by Epstein-Barr virus encephalitis. AJNR Am J Neuroradiol 2006;27:1447-9.

8. Rafailidis PI, Mavros MN, Kapaskelis A, Falagas ME. Antiviral treatment for severe EBV infections in apparently immunocompetent patients. J Clin Virol 2010;49:151-7.

9. Lim JA, Byun JI, Lee ST, Jung KH, Kim YS, Kim JM, et al. Epstein-Barr virus brainstem encephalitis with anti-N-methyl-D-aspartate receptor antibodies. J Korean Neurol Assoc 2013;31:199202.

10. Abul-Kasim K, Palm L, Maly P, Sundgren PC. The neuroanatomic localization of Epstein-Barr virus encephalitis may be a predictive factor for its clinical outcome: a case report and review of 100 cases in 28 reports. J Child Neurol 2009;24:720-6.

11. Kasper D, Fauci A, Hauser S, Longo D, Jameson JL, Loscalzo J.
Harrison's principles of internal medicine, 19th ed.

12. Sonneville R, Klein I, de Broucker T, Wolff M. Post-infectious encephalitis in adults: diagnosis and management. J Infect 2009;58: 321-8.

13. Reindl M, Di Pauli F, Rostasy K, Berger T. The spectrum of MOG autoantibody-associated demyelinating diseases. Nat Rev Neurol 2013;9:455-61.

14. Schwarz S, Mohr A, Knauth M, Wildemann B, Storch-Hagenlocher B. Acute disseminated encephalomyelitis: a follow-up study of 40 adult patients. Neurology 2001;56:1313-8.

15. Mader I, Stock KW, Ettlin T, Probst A. Acute disseminated encephalomyelitis: MR and CT features. AJNR Am J Neuroradiol 1996;17:104-9. 